\title{
Anatomic Relationship between the Impacted Mandibular Canine and the Mental Nerve: A Case Report
}

\author{
Shivendra Singh ${ }^{1}$, Meghna Kumar ${ }^{2}$, Nishant Singh ${ }^{3}$, Ankit Singla ${ }^{4}$, Gaurav Rai ${ }^{5}$, Kirti Prakash ${ }^{6}$ \\ ${ }^{1}$ Assistant Professor, Department of Oral and Maxillofacial Surgery, Rama Dental College, Hospital and Research Centre, \\ Kanpur, ${ }^{2}$ Post-Graduate Student, Department of Oral and Maxillofacial Surgery, Rama Dental College, Hospital and Research \\ Centre, Kanpur, ${ }^{3}$ Professor, Department of Oral and Maxillofacial Surgery, Rama Dental College, Hospital and Research \\ Centre, Kanpur, ${ }^{4}$ Post-Graduate Student, Department of Oral and Maxillofacial Surgery, Rama Dental College, Hospital and \\ Research Centre, Kanpur, ${ }^{5}$ Post-Graduate Student, Department of Oral and Maxillofacial Surgery, Rama Dental College, \\ Hospital and Research Centre, Kanpur, ${ }^{6}$ Post-Graduate Student, Department of Oral and Maxillofacial Surgery, Rama Dental \\ College, Hospital and Research Centre, Kanpur, India
}

Corresponding author: Dr Meghna Kumar (MDS), Post-Graduate Student, Department of Oral and Maxillofacial Surgery, Rama Dental College, Hospital and Research Centre, Kanpur, India

DOI: http://dx.doi.org/10.21276/ijcmsr.2020.5.1.14

(c) BY-NC-ND

How to cite this article: Shivendra Singh, Meghna Kumar, Nishant Singh, Ankit Singla, Gaurav Rai, Kirti Prakash. Anatomic relationship between the impacted mandibular canine and the mental nerve: a case report. International Journal of Contemporary Medicine Surgery and Radiology. 2020;5(1):A56-A58.

\section{A B S T R A C T}

Introduction: Impacted teeth can be defined as those that do not erupt at the normal age of eruption and remain embedded in the maxilla or mandible where they are either partially or completely surrounded by bone or soft tissues. Clinically, impacted teeth are seen in about $20 \%$ of the population. Third molars are the most commonly impacted teeth, followed by the maxillary canines and the mandibular bicuspids.

Case report: A 55-year old male reported to the Department of Oral and Maxillofacial Surgery, Rama Dental College; complaining of pain and swelling in the lower left anterior region of the jaw since 1 week. An ortho-pantomogram of the patient revealed an impacted distoangular 33, at Level $\mathrm{C}$ with respect to 34 . The patient was prescribed oral antibiotics and analgesics for three days to alleviate pain and swelling. The impacted tooth(33) was then surgically extracted under local anesthesia. The mental foramen was located apical to 34. The patient was recalled after a week for suture removal. The healing was uneventful. No paresthesia of the left side of lower lip, left gingiva or left buccal mucosa was observed.

Conclusion: This case report emphasizes the need for careful radiographic assessment of the mandibular canal and foramina pre-operatively and careful sub-periosteal dissection to avoid inadvertent damage to the mental nerve during extraction of impacted mandibular canine leading to paresthesia of the lower lip, mandibular labial gingiva and chin.

Keywords: Canine Impaction, Impaction, Mental Nerve, Trans-Alveolar Extraction

\section{INTRODUCTION}

Impacted teeth can be defined as those that have not erupted into occlusion according to chronological age due to obstruction by bone or soft tissue. Impaction of teeth is clinically seen commonly in about $20 \%$ of the population. ${ }^{1}$ The frequency of impaction in Mongoloid races has been found to be 2.5 times more than in Caucasians. ${ }^{2}$ Third molars are the most commonly impacted teeth(mainly in the mandible) followed by maxillary canines and the mandibular premolars. ${ }^{3}$ Impacted teeth must be diagnosed and treated promptly, as they may lead to formation of pathological cysts. Impaction of maxillary canines has an incidence of between 0.8 to $2.3 \% .^{4}$ The occurrence of impaction and/or noneruption of mandibular canines is unusual, with prevalence rates from 0.05 to $0.4 \% .{ }^{5}$ Several etiological factors have been linked with the impaction of a mandibular canine, such as an eruption disturbance caused by an anomaly of the canine germ itself. ${ }^{6}$ Trauma, pathological conditions (odontomes, cyst), crowding, and early loss of primary canines and mandibular fractures may also be a causative factor. ${ }^{7}$

Often, impacted teeth may be retained while still asymptomatic. $^{8}$ However, Bishara et al. suggested the following sequelae of canine impaction ${ }^{9,10}$ :

- Movement of the impacted tooth to a labial or lingual position

- Migration of the adjacent teeth and reduction in arch length

- Root resorption of the impacted tooth as well as the adjacent teeth

- Inflammation associated with partial eruption causing pain and trismus

- Pain radiation to the temporo-mandibular jaw or the temporal region

The mental nerve, arising from the mental foramen present in the canine-premolar region provides sensory innervation to the chin, labial gingival and lower lip. Therefore, the nerve should be isolated and protected during all surgical 
procedures in the symphysis or parasymphysis region to avoid any potential paresthesia.

\section{CASE REPORT}

Initial examination: A 55-year old male reported to the Department of Oral and Maxillofacial Surgery, Rama Dental College, complaining of pain and swelling in the lower left anterior region of the jaw since 1 week. An ortho-pantomogram of the patient revealed an impacted distoangular 33, at Level C with respect to 34 (Image 1). No significant medical history was found, and no other extraoral or intraoral abnormality was detected.The patient was prescribed antibiotics and analgesics to alleviate the symptoms, and was recalled after two days.

Treatment: The patient was prepared and draped according to standard protocol. The inferior alveolar nerve block and the

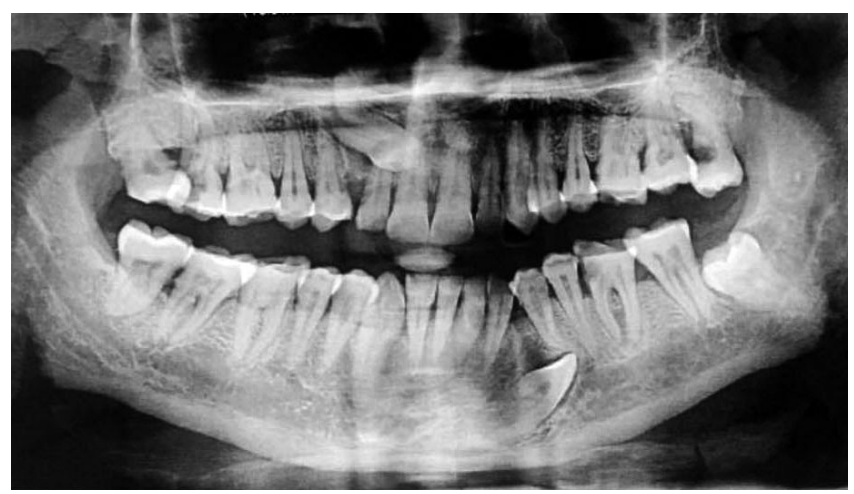

Image-1: Ortho-pantomogram revealing the offending tooth to be an impacted 33 at Level $\mathrm{C}$ with respect to 34

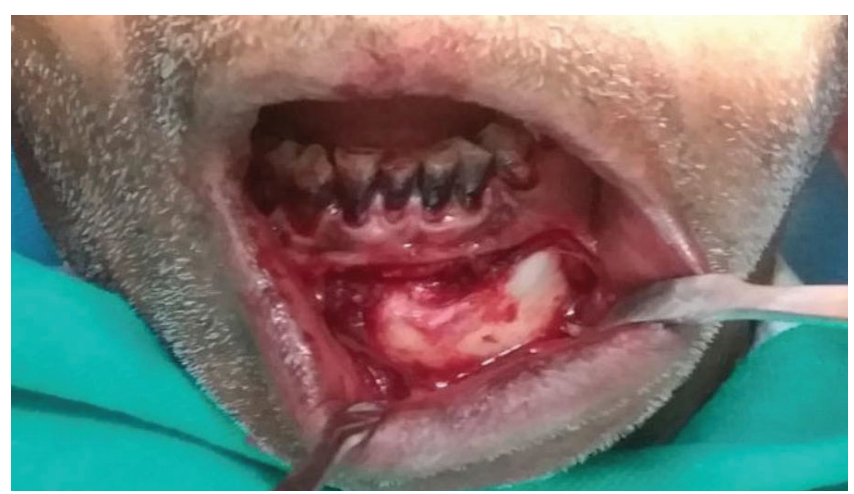

Image-2: Sub-periosteal dissection done after giving a vestibular incision extending from 41 to 35

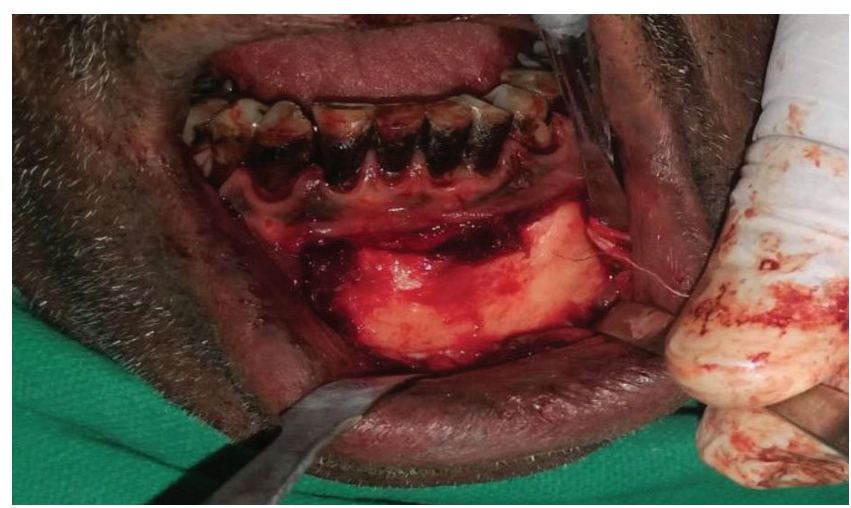

Image-3: The mental foramen located apical to 34

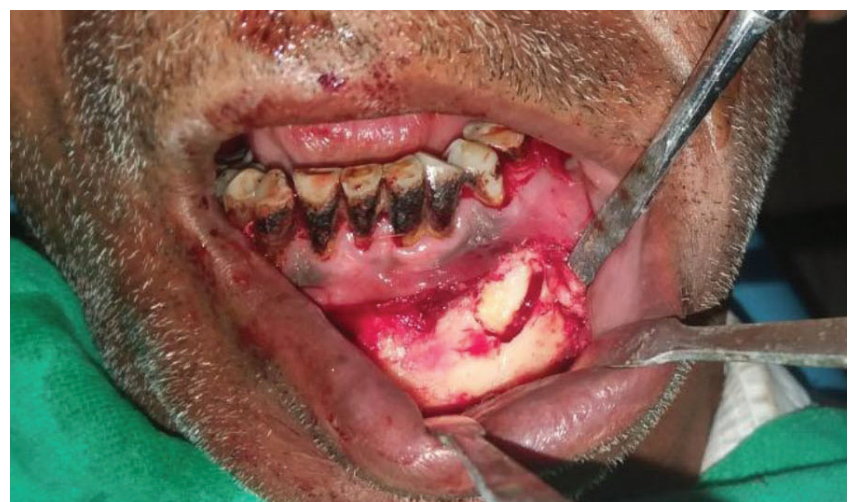

Image-4: Exposure of impacted 33

lingual nerve block was administered bilaterally. Additionally, an infiltration nerve block was given between 32 and 35 to minimize bleeding at the operation site.

A vestibular degloving incision was given extending from 41 to 35. Careful sub-periosteal dissection was done both superiorly and inferiorly to expose the crown of 33 and the inferior border of the mandible respectively, while preserving the mental nerve (Image 2). The mental foramen was located apical to 34 (Image 3). A stainless steel round bur was used to completely expose the crown of the impacted canine and the root up to its upper one third (Image 4). The crown was sectioned using a no.702 stainless steel bur. The crown and root were then removed separately.

The bony socket was irrigated with $5 \%$ betadine solution and the defect was closed with simple, interrupted sutures using 3-0 silk. A pressure dressing with gauze and dynaplast was applied and the patient was prescribed a course of Amoxicillin+Potassium Clavulanate (625mg) TID, Metronidazole (400mg) TID, Aceclofenac + Serratiopeptidase + Paracetamol (100mg+15mg+325mg) $\mathrm{BD}$, and Pantoprazole (40mg) OD for 5 days.

Follow-up: The patient was recalled after seven days for suture removal. The healing was uneventful. No paresthesia of the left side of lower lip, left gingiva or left buccal mucosa was observed.

\section{DISCUSSION}

Mandibular canine impaction is considered to be less common phenomenon than maxillary canine impaction, and only a few studies recording its frequency can be found in literature. ${ }^{16}$ Grover and Lorton found only 11 impacted canines $(0.22 \%)$ in the mandible in 5000 individuals. Chu et $\mathrm{al}^{17}$ reported five mandibular impacted canine $(0.07 \%)$ teeth in 7486 patients. Rohrer ${ }^{18}$ examined 3,000 patients and found 62 impacted maxillary canines $(2.06 \%)$ as compared to only three impacted mandibular canines $(0.1 \%)$.

Multiple etiologies have been described which can lead to failure of eruption of teeth. Most surgeons are of the opinion that these etiologies may include a pathological condition, inflammation, interference of prosthetic devices, disturbances of the dentition, pain, and ectopic eruption. Etiologies specific to the unerupted mandibular canine include insufficient space, supernumerary teeth, premature loss of the primary teeth, retained deciduous canine, excessive crown length, genetic factors, functional disturbances of the endocrine 
system, tumours, cystic lesions and traumatic accidents. Impacted mandibular canines have been reported to be more commonly located on the labial aspect of the dental arch than the maxillary canines, and the removal of impacted teeth routinely involves an intraoral surgical approach. ${ }^{19}$

Impacted mandibular canines can be classified based on angulations and depths of the involved teeth. With respect to angulation, impacted mandibular canines can be classified as mesioangular, distoangular, vertical, or horizontal.

With respect to depth, impactions can be classified as Level A, Level B, and Level C:

Level A. The crown of the impacted canine tooth is at the cervical line of the adjacent teeth.

Level B. The crown of the impacted canine tooth is between the cervical line and root apices of the adjacent teeth.

Level C. The crown of the impacted canines is beneath the root apices of the adjacent teeth.

Mental Nerve: The mental nerve arises from the inferior alveolar nerve within the inferior alveolar canal, which is a branch of the mandibular division of the trigeminal nerve. It divides into three branches as it leaves the mental foramen the mental branch which supplies the chin, the labial branch which supplies the lower lip and the gingival branch which supplies the gingival. ${ }^{11}$ However, several anatomic variations of the branching patterns of the mental nerve have been reported to date.

Several authors have reported the presence of accessory mental foramina. Sawyer et al. studied the occurrence of accessory mental foramina in four ethnic groups. ${ }^{12}$ The frequency of accessory foramina was found to be highest in Nazca Indians (9\%) and lowest in Asian Indians (1.5\%). However, another study on Asian Indian mandibles reports the incidence to be much higher $(6.6 \%) .{ }^{13}$ The location of the mental foramen has also been proven to show variations. Fishel et al. showed that, while in $70.4 \%$ of the population, the mental foramen is located between the apices of two premolars, others showed vertical variations. ${ }^{14}$ Variations in shape of the mental foramina have also been reported, with the round shape found to be more common by some authors ${ }^{15}$ and the oval shape more common by others. ${ }^{16}$

\section{CONCLUSION}

The mandibular impacted canine is usually associated in close proximity with the apex of the mandibular premolars, and thus the mental nerve and mental foramen. This case report emphasizes on the need for careful preoperative radiographic assessment of the inferior alveolar canal and the mental foramina followed by careful sub-periosteal dissection to avoid inadvertent damage to the mental nerve leading to paresthesia of the lower lip, mandibular labial gingiva and chin.

\section{REFERENCES}

1. Attasalo K, Lehtinen R, OksalaE. An orthopantomographic study of prevalence of impacted teeth. Int J Oral Surg 1972; 1(1): 117- 20.

2. Kramer RM, Williams AC. The incidence of impacted teeth. Oral Surg Oral Med Oral Pathol 1970;29(3):23741.
3. Grover PS, Lorton L. The incidence of unerupted permanent teeth and related clinical cases. Oral Surg Oral Med Oral Pathol 1985; 59(5):420-5.

4. Thilander B, Myrberg N. The prevalence of malocclusion in Swedish schoolchildren. Scan J Dent Res 1973;81(2):12-20

5. Brown LH, Berrkman S, Cohen D, Kaplan AL, Rosenberg MA. A radiological study of the frequency and distribution of impacted teeth. J Dent Assoc S Afr 1982; 37(4): 627-30.

6. Andreasen JO. The impacted mandibular canine. In AndreassenJO, Petersen JK, Laskin DM (eds). Textbook and Color Atlas of Tooth Impaction, 1st edn. Copenhagen: Munksgaard; 1995. Pp167, 175.

7. Morning P. Impacted teeth in relation to odontomas. Int J Oral Surg1980;9(6):81-91.

8. Yamaoka M, Furusawa K, FujimotoK, Uematsu T. Completely impacted teeth in dentate andedentulous jaws. Aust Dent J 1996: 41(5):169-72.

9. Bishara SE, Kommer DD, McNeil MH, Montagano LN, Oesterle LJ, Youngquist W. Management ofimpacted canines. Am J Orthod 1976: 69(1):371-87.

10. Bishara SE. Impacted maxillary canines. Am J OrthodDentofacOrthop 1992: 101(3):159-71.

11. Standring S, editor. Gray's Anatomy: The Anatomical Basis of Clinical Practice. 39th ed. London: Elsevier Churchill Livingstone; 2005. p. 601-2.

12. Sawyer DR, Kiely ML, Pyle MA. The frequency of accessory mental foramina in four ethnic groups. Arch Oral Biol 1998;43(2):417-20.

13. Gupta S, Soni JS. Study of anatomical variations and incidence of mental foramen and accessory mental foramen in dry human mandibles. Nat J Med Res 2012;2(1):28-30.

14. Fishel D, Buchner A, Hershkowith A, Kaffe I. Roentgenologic study of the mental foramen. Oral Surg Oral Med Oral Pathol 1976;41(6):682-6.

15. Al-Khateeb T, Al-HadiHamasha A, Ababneh KT. Position of the mental foramen in a northern regional Jordanian population. SurgRadiolAnat 2007;29(4):231-7.

16. Oliveira Junior EM, Araiyo AL, Da Silva CM, Sousa-Rodrigues CF, Lima FJ. Morphological and morphometric study of mental foramen on the M-CP-18 Jiachenjiang point. Int J Morphol (Temuco) 2009;27(3):231-8.

17. Camilleri S, Scerri E. Transmigration of mandibular canines-A review of the literature and a report of five cases. Angle Orthod 2003: 73(5):753-762.

18. Grover PS, Lorton L. The incidence of unerupted permanent teeth and related clinical cases. OralSurg Oral Med Oral Pathol 1985: 59(4):420-425.

19. Chu FCS, Li TKL, Lui VKB, Newsome PRH, Chow RLK, Cheung LK. Prevalence of impacted teeth and associated pathologies - a radiographic study of the Hong Kong Chinese population. Hong Kong Med J 2003: 9(2):158-163.

Source of Support: Nil; Conflict of Interest: None

Submitted: 02-12-2019; Accepted: 30-12-2019; Published online: 27-01-2020 